\title{
A Questionnaire based Examination of Community Networks
}

\author{
Jeroen Avonts, Bart Braem, Chris Blondia \\ Department of Mathematics and Computer Science \\ University of Antwerp - iMinds - PATS Research Group \\ Middelheimlaan 1, B-2020, Antwerp, Belgium \\ Email: \{jeroen.avonts,bart.braem,chris.blondia\}@uantwerpen.be
}

\begin{abstract}
There is a variety of community networks out there. In this paper we try to shed a light on the community network landscape by presenting the results of a questionnaire filled in by different community network organisations around the world. We demonstrate the variety of infrastructure used and the different management approaches of the network within those communities. We also show the common challenges of the communities and provide pointers to tackle those challenges.
\end{abstract}

\section{INTRODUCTION}

When a network infrastructure is extended over multiple locations in a neighbourhood and the community is allowed to access this network, we call this network a community network[1]. Community networks are built for a variety of reasons. Some provide internet access to remote locations where the cost versus gain is too high for commercial providers. Others find their existence in the curiosity of the technical possibilities of networks. There are community networks built out of a few single nodes to networks with thousands of different nodes. Although the variety in community network setups is large, most of the communities have common goals and share the same challenges. Our goal is to show the differences between those community networks and especially indexing their common challenges.

In this paper we present the results of a questionnaire sent to different communities network organisations. With the results we try to illuminate the community network landscape; we demonstrate the variety of infrastructure used, the variety in technical approaches and the variety in management. We show common patterns in their challenges such as funding, finding volunteers and maintaining the network. By indexing those common challenges, communities can learn from other communities and improve their own network.

\section{APPROACH}

We set up a questionnaire of 35 questions (see appendix) related to the infrastructure, the management and the challenges of community networks. We used Google Docs to create the questionnaire. This questionnaire was distributed via the IS4CWN[2] and open mailing lists. The total number of filled entries was twenty with one duplicate entry for a certain community network. As such, a total of nineteen unique community networks entries were analysed. We preferred open questions over multiple choice to allow additional comments in the answer. This additional information helped us to interpret the answers correctly. We compared the answers and present the findings in this paper.

The questions were divided in six categories.

General information: The information retrieved in this category allows us to uniquely identify and geographically locate the community network. We ask whether the community networks have a website that can be used to find additional information about the network.

Network organisation: The network organisation category gives us insight in the number 
and activity of subscribers, the legal entity of the network and connections to other community networks.

Infrastructure: The answers in the infrastructure category give us a sense of the scale, and the software and hardware that is used in the network.

Maintenance: Every community network has its own workflow to add and maintain nodes and links in the network. With the questions in the maintenance category we try to get a view on how network nodes are added and maintained, and who is responsible for this installation and maintenance.

Legal and financial aspects: The legal and financial category gives us the type of subscription the users agree with and the funding of the network.

Closing questions: The closing questions ask for the largest overall challenge and whether the answers given are allowed to be published.

We keep encouraging community networks to fill in the questionnaire or modify them when answers are outdated. These living data are shared with the communities. This allows the communities to compare their own methods to those of others and to find solutions in other networks for certain challenges they might face.

\section{RESULTS}

Nineteen unique communities from Africa, $\mathrm{Eu}-$ rope, North and South America filled in the questionnaire. This is about $30 \%$ of the active community networks known to us. Although we obtained a decent geographically spread, networks from Asia and Australia are missing. This section provides the statistics of the questionnaire for those $30 \%$ of active networks. We indicate when the results are based on fewer entries than all the nineteen because not all the answers were filled in by the communities. The results are further discussed in section IV.

\section{A. General Information}

Nine networks are located in North America; seven of them in the USA (Personal Telco Project, Hot Mesh, Project Byzantium, Mount Pleasant Community Wireless Network, WasabiNet, Cass Corridor Network, Red Hook Initative WiFi) and two in Canada (Réseau Libre, Kuhkenah Network). Six are located in Europe; one in each of the following countries: Greece (Athens Wireless Metropolitan Network), Slovenia (Wlan Slovenija), Italy (Ninux), Catalonia in Spain (guifinet), Austria (Funkfeuer) and the Netherlands (Wireless Leiden). Three communities are in South America: one in Chile (LicaNet), one in Argentina (DeltaLibre) and one in Colombia (Bogota Mesh). Finally, one network is located in South Africa $(S W U G)$ in Africa.

All the community networks except one, started in or after the year 2000. There are peaks in 2001 and 2011. The oldest network is Kuhkenah Network and was founded in 1994.

\section{B. Network Organisation}

One of the major goals of community networks is to provide network access to the users in the community. The number of active users can be tracked with some sort of subscription model. We provide the results concerning the subscription model and the number of subscriptions. We also have a look at the legal entity of the community and inter-community-network connections.

Users subscription: Ten or 53\% of the communities use a subscription method. The remaining nine $(47 \%)$ have little to no knowledge of the number of subscribers in the network or do not use any method of subscription. Of the ten communities that use a subscription method, eight (80\%) have between 10 to 1000 subscriptions with a mean of around 170 subscribed users; two (20\%) have more than 10000 subscribers.

Active users: Three or $16 \%$ of the community networks do not know or do not track the average number of active users. Of the remaining sixteen (84\%) community networks, fifteen (94\%) have 
an average number of active users ranging from 10 to 5000 with the mean around 640 users. One community network has more than 50000 users. The maximum number of active users on a single moment is unknown or not tracked by five $(26 \%)$ community networks. Of the fourteen (74\%) remaining, thirteen $(93 \%)$ have a maximum amount of active users between 10 and 1000 with the mean around 220 users and one network has more than 50000 users.

Legal entity: There is a legal entity (non-profit organisation, $\mathrm{NGO}, \ldots)$ in ten $(53 \%)$ community networks.

Interconnections: Three or $16 \%$ of the community networks have active connections with other community networks. The CONFINE[3] project interconnects guifi.net, Funkfeuer and Athens Wireless Metropolitan Network (AWMN). The community network Wlan Slovenija is interconnected with Funkfeuer and NEDWireless.

\section{Infrastructure}

The network nodes of a community network are often a mix of different hardware and software. Knowing the number of nodes, their locations, their hardware components and their software configurations, can be challenging. We take a closer look at this specific information and how it is managed.

Node Database: We asked how the infrastructure of the community network is documented. Three (16\%) community networks do not use any kind of database to keep track of their nodes and links in the network. Of the sixteen $(84 \%)$ that do have some kind of database, thirteen $(81 \%)$ allow public access, often via a web interface, to this node database. For the other three (19\%) this database is held private. These results are depicted in Figure 1 . Updating the database of the community network, such as adding nodes and updating link information, is done exclusive manually by ten $(62 \%)$ of the sixteen community networks community networks that have a node database. For four or $25 \%$ of those community networks the database is updated automatically. A combination

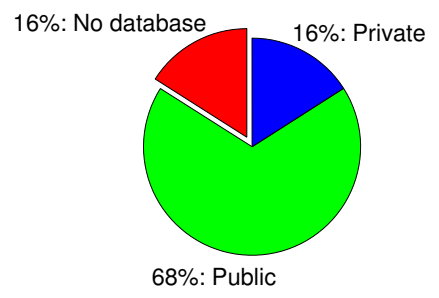

Fig. 1. Access policy to the community network infrastructure database.

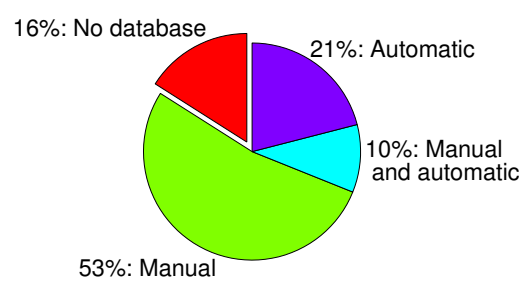

Fig. 2. Updating method of the community network infrastructure database.

of manual input with automatic updates of the links is used in two of those community networks (12\%). These results are shown in Figure 2.

Nodes: The number of nodes in the network is unknown for one community network. For sixteen $(89 \%)$ of the remaining eighteen community networks, the number of nodes ranges between 5 to 500 with the mean around 84 . Two community networks have more than 1000 nodes with one more than 10000 nodes. The distribution of the number of nodes on a log scale is depicted in Figure 3.

Links: For five community networks the number of links between the nodes is unknown. Of the remaining fourteen, eleven $(79 \%)$ have a number of links ranging from 2 to 300 with the mean around 67. Three community networks have a number of links exceeding 1000 with one more than 10000 links. Figure 4 depicts the distribution of the number of links on a log scale.

Hardware: The different community networks often use a specific hardware configuration. We provide the top three of hardware used. For one 


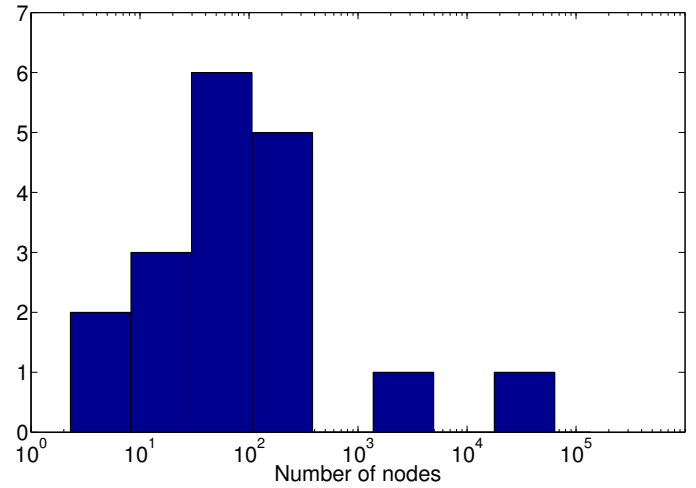

Fig. 3. Histogram of the number of nodes in the community networks.

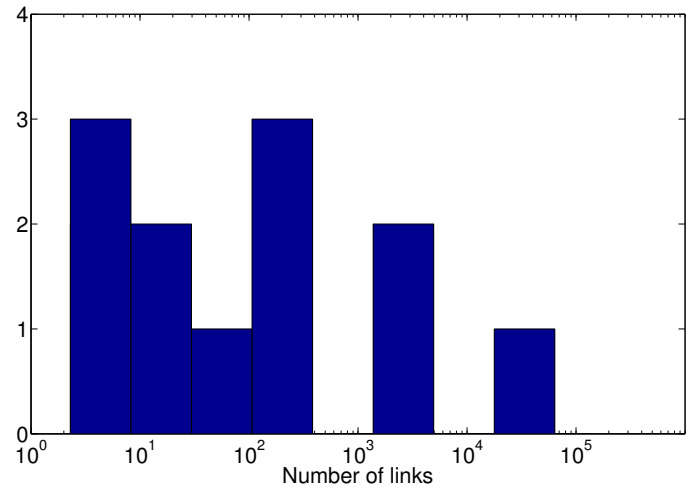

Fig. 4. Histogram of the number of links in the community networks

community network the answer was unclear and is for this question, left out in the statistics. The following top three is based on eighteen community networks. In first place, used in ten (56\%) community networks, is the Ubiquiti[4] hardware. In second and third place are MikroTik[5] and Atheros [6] both mentioned in three (17\%) community networks. All three community networks that use MikroTik hardware also mentioned using Ubiquiti hardware. And Ubiquiti hardware is also used in one community network of the group that uses Atheros. Figure 5 depicts these results. All nineteen community networks use some type of wireless technology complemented with optical fiber, xDSL or mobile infrastructure. The 802.11n technology is used in nine $(47 \%)$ community networks. We take a closer look at the wireless hard-

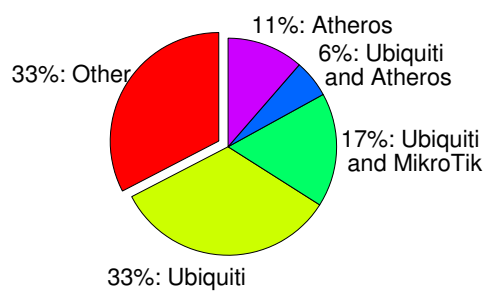

Fig. 5. Hardware used in the community networks.

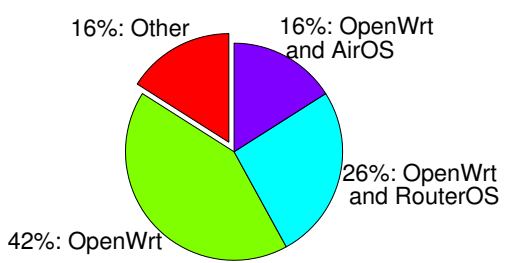

Fig. 6. Operating systems used in the community networks.

ware vendors used in the community networks. The following results are based on the fifteen community networks that answered this question. The top consists of, in first place, Ubiquiti used by eight $(53 \%)$ community networks and in second place, TP-link used in three $(20 \%)$ networks. In two (13\%) community networks both TP-link and Ubiquiti hardware are used. The remaining six $(40 \%)$ use a variety of other hardware vendors.

Software: For sixteen (84\%) community networks, the preferred operating system used on the hardware is OpenWrt[7]. Other linux distros are mentioned in five $(26 \%)$ community networks. In three (16\%) community networks RouterOS[8] used by MikroTik, and also in three (16\%) AirOS[9] used by Ubiquiti, is mentioned. RouterOS and OpenWrt are both mentioned in five $(26 \%)$ community network's answers. These results are shown in Figure 6. We had a more detailed look at the software, protocol, or algorithms used for the routing in the community networks. In first place, used by ten (53\%) community networks, comes OLSR[10] as the main routing protocol. Used in three (16\%) community networks and in second place is $B G P[11]$. Third 


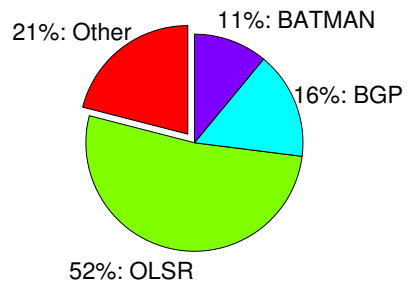

Fig. 7. Routing protocols used in the community networks.

place and used in two (11\%) community network, is BATMAN[12]. The remaining four (21\%) use some different type of routing protocol. Figure 7 depicts these results.

User access: In eight (42\%) community networks there is no separation between the end user's access network and the backbone network interconnecting the end user's access network. In the remaining eleven (58\%) community networks, the end user is separated from the backbone network.

\section{Maintenance}

Community networks change and grow in time. Nodes and links might fail and need maintenance, also new nodes and links can be introduced. We take a look at the request-installation-maintenance chain of new nodes and links.

Nodes: New nodes in the network can be requested by anybody in fourteen (74\%) community networks. In four $(21 \%)$ community networks, a dedicated team decides on new node installation and in one community network, the city hall dictates the installation of new nodes. The installation of those new nodes can in nine $(47 \%)$ community networks, be done by anybody. A dedicated team does the installation in eight $(42 \%)$ community networks and for the remaining two (11\%) either the IT-department of the city hall or paid professionals install the nodes. In eleven (58\%) community networks the owner of the node is responsible for the maintenance and in ten $(53 \%)$ a dedicated team maintains the nodes. For four ( $21 \%)$ community networks both the owner and a dedicated team are mentioned. Paid professionals are mentioned in two (11\%) community networks and in one community network, the IT-department of the city hall is responsible for the maintenance.

Links: The decision for new links between the network nodes can in thirteen (68\%) community networks, be made by anybody. A dedicated team decides in five $(26 \%)$ community networks on new links and in one network, the city hall makes this decision. The installation and maintenance of links was not answered by one community network. The following results are based on the remaining eighteen community networks. The activation of the new links can in fifteen $(78 \%)$ community networks, be done by anybody. In three (17\%) community networks this activation is done by a dedicated team and in one community network the activation is done by the IT-department of the city hall. The maintenance is for ten $(56 \%)$ community networks done by the owners of the links and for seven $(39 \%)$ a dedicated team maintains the links. One community network also mentioned paid professionals to aid the owners. Another community network has the IT-department of the city hall take care of the maintenance.

\section{E. Legal and financial aspects}

For the best interest of the network each user should cooperate in the network and might be restricted by some rules. We examine whether users have to sign an agreement to use the network. Setting up a community network is often done by volunteers and with limited budget for the node hardware. We also have a look at how the community networks get funded.

Agreement: Five (26\%) community networks have some kind of agreement to be signed before using the network. This agreement is mainly an informal one. The community networks that do not use a signed agreement often have a website indicating the restrictions and rules when using the network.

Funding: The main source of funding comes from the community members themselves; this is the case for eleven $(58 \%)$ community networks. 
Seven (37\%) mentioned the government as a source of funding where three $(43 \%)$ of those seven have the government as the only funder. In four $(21 \%)$ community networks, donations are the main source of funding where two (50\%) of them depend solely on donations. Businesses partners are mentioned in two (11\%) community networks.

\section{F. Challenges}

Each community network has its own challenges depending on the region, size, location, etc. of the network. We list the top three challenges for the different categories.

Organisational: The main organisational challenge for six $(32 \%)$ community networks, consists of finding funds to maintain and expand the community network. For six $(32 \%)$ community networks finding and keeping volunteers is considered the most organisational challenging. And for five $(26 \%)$ community networks growth of the network and the community is one of the main challenges.

Infrastructure: For six (32\%) community networks, creating reliable wifi links is the main infrastructural challenge. This is followed by five (26\%) community networks that mentioned decent node locations as main challenge. The remaining community networks listed other items such as IPv6[13], hardware failure, deploying fiber and decent uplinks as main infrastructural challenge.

Maintenance: This question was not filled in by one community, results are based on eighteen community networks. For nine (50\%) community networks, basic node maintenance is the most challenging. This includes monitoring, software updates and configuration. Finding volunteers to maintain the nodes, often only reachable at office hours, is for seven $(39 \%)$ the main maintenance challenge. And on the third place for two (11\%) community networks, physical access to the nodes proves to be the main challenge.

General: Two community networks left this question blank. The results are based on seventeen community networks. The main overall challenge for six $(35 \%)$ community networks is finding the necessary human resources. This includes finding volunteers to install and maintain nodes as well as volunteers handling the administration and keeping the volunteers involved in the long-term. Three (18\%) community networks are looking for a sustainable model for funding the further expansion of the network and of the community. Another three $(18 \%)$ community networks mentioned that conveying the message of community networks is the main overall challenge.

\section{ANALYSIS OF THE RESULTS}

In this section we discuss some interesting patterns from the results.

\section{A. Network Information}

Although most of the community networks have some sort of database with the node information, most of the community networks do not have a clear overview of their actual network. The database is often manually filled, prone to human errors and mostly outdated. Automation of crawling the network and gathering data of the network can help to get the most correct view on the network. Storing node dependent information on the node itself removes the necessary step of mapping some node id onto the manually filled database to get, for example, the physical location of a node or device.

The time series of data obtained by crawling is also valuable to research the construction of community networks[14], [15], [16]. Even small community networks can benefit from the crawled information when they start expanding. The crawled data also provides insight in the condition of the network over time. Node or link failures can easily be discovered by comparing older crawling data with the new one. Those differences can be used to trigger node or link maintenance alerts.

In addition to monitoring the state of the nodes and links in the network, monitoring the flows of traffic through the network can help to identify bottlenecks. Finding new locations can be 
prioritised using this network flow information. Tools for traffic flow monitoring are available, for example, the RouterOS has the MikroTik TrafficFlow[17] functionality built in.

\section{B. Knowledge}

Each community network has its own workflow of setting up new nodes. For example, certain configurations using wireless distribution system (WDS) are set up to establish a link, or the IPsubnets are assigned according to some rules. It is important to document these steps within the workflow and keep track of exceptional configurations. We propose to keep this in a living document, such as a wiki, for easy editing and sharing. The more documentation new volunteers have when entering the community, the more knowledge they have to maintain their own devices. It also removes the single point of failure of one person knowing how certain sites are set up.

We also propose to let the users of the community sign or at least agree with a set of written regulations. This way, the user is provided with their rights and obligations when entering the network. This document can be used to solve disputes at a later stage.

\section{Funding}

The largest challenge throughout all categories is finding the budget to run the community network. This budget often comes from the community members themselves, yet some community networks have successfully negotiated funding with their government. A first step to make the negotiations with the government easier, is creating a legal entity. This was done by all community networks that get funded by their local government. The smaller the government is and the more local they operate, the more willing they seem to be to fund expansion of the network as a service for the community.

Another option is to cooperate with businesses. Especially looking for a win-win situation between businesses and the community. For exam- ple, a company wants to have a dedicated link between two of its distribution sites. The community could set up this connection and use the node location for additional hops for the community network, separated from the dedicated link of the company.

Some community networks look for or have a sustainable model. This could be, for example, providing additional services for paying users; services such as higher bandwidth, QoS, etc.. It is beneficial for all community networks to share their model with the community networks looking for a sustainable funding model.

\section{Volunteers}

Although some community networks use paid professionals for installation and maintenance, the majority of the community networks is administrated by volunteers. Because community networks often start with a few technically skilled persons who take on the challenge of creating a network in their neighbourhood, it might seem that technically skills are obliged when joining the community network organisation. Yet besides the obvious technical aspects of a community network, there are also non-technical aspects, such as administrational and promotional aspects, and even bookkeeping. Having volunteers for these type of tasks allows every volunteer to focus on their strengths; this in turn keeps the volunteers motivated in the long run.

As mentioned in section IV-B, to engage new volunteers as quickly as possible, the volunteers need to have access to decent documentation about the network. This documentation should be both technically in depth as well as high level and abstract. By providing this documentation as a living document, new information is easily added and updated by the volunteers. This way, when a volunteer leaves the community, another volunteer can easily take over his or her task.

\section{CONClUSiON}

In this paper we tried to get a view of the landscape of community networks. We set up a 
questionnaire and sent it to different communities network organisations. We presented the results of the answers for the different categories related to the infrastructure, the technical aspects and the management of community networks. Further on, we analysed the answers and isolated a number of common major challenges of community networks. Finally, we provided pointers to tackle those challenges.

\section{ACKNOWLEDGMENT}

This work is supported by the CONFINE Integrated Project 288535.

\section{QUESTIONNAIRE}

\section{General information:}

- How is your community network called?

- Do you have a website for your network?

- In which country/countries is your community network located?

- In which region(s) is your community network located?

- When was your network started?

Network organisation:

- How many users are "subscribed" to your network?

- How many users are active on average?

- What was the maximum number of active users ever?

- Is there a legal entity behind your network? If yes, which type (NGO, foundation, business, ...)?

- Are you connected to other community networks? If yes, which ones and how?

- Which is your largest organisational challenge?

Infrastructure:

- Do you have a node database? And is it publicly accessible?

- How is the node database updated?

- About how many nodes are installed in your network?

- About how many links are installed in your network?

- Which is the dominant hardware platform for nodes?

- Which is the dominant OS used in your network? Are multiple OSes being combined?

- Which is the dominant communication technology used in your network? Are multiple technologies being combined?

- Which is the dominant wireless technology platform or vendor?

- Which routing protocol is used in the network?

- Do end users have access to the backbone network? Or do they only access the network over access points?

- Which is your largest infrastructure challenge?

Maintenance:

- Who can install new nodes in the network?

- Who can install new links in the network?

- Who maintains the network nodes?

- Who maintains the links?

- Who proposes and decides on new links?

- Could you describe the process of adding a new node, from request to connection to the network?

- What is your largest maintenance challenge?

\section{Legal and financial aspects:}

- Do you require users to sign a peering agreement? Or another contract-like document?

- Which is the primary source of funding for your network?

\section{Closing questions:}

- Which is your largest overall challenge?

- Do you have other comments or questions to the authors of this survey? Also, can we publish your answers?

- Can we contact you for more questions? If yes, please provide an e-mail address.

\section{REFERENCES}

[1] Bart Braem, Chris Blondia, Christoph Barz, Henning Rogge, Felix Freitag, Leandro Navarro, Joseph Bonicioli, Stavros Papathanasiou, Pau Escrich, Roger Baig Viñas, Aaron L. Kaplan, Axel Neumann, Ivan Vilata i Balaguer, Blaine Tatum, and Malcolm Matson. A case for research with and on community networks. SIGCOMM Comput. Commun. Rev., 43(3):68-73, July 2013.

[2] International Summit for Community Wireless Networks. http://wirelesssummit.org/.

[3] CONFINE: Community networks testbed for the future internet. http://confine-project.eu/.

[4] Ubiquiti networks. http://www.ubnt.com/.

[5] MikroTik routers and wireless. http://www.mikrotik.com/.

[6] Qualcomm Atheros. http://www.atheros.com/.

[7] The OpenWrt linux distro. https://openwrt.org/.

[8] RouterOS, the operating system of RouterBOARD. http://www.mikrotik.com/software.html.

[9] AirOS, the operating system of Ubiquiti.

[10] T. Clausen and P. Jacquet. Optimized Link State Routing protocol (olsr), 2003.

[11] Y. Rekhter, T. Li, and Hares S. A Border Gateway Protocol 4, 2006.

[12] The Better Approach To Mobile Adhoc Networking. http://www.open-mesh.org/projects/open-mesh/wiki.

[13] S. Deering, Cisco, and Hinden R. Internet Protocol, Version 6, 1998.

[14] Llorenç Cerdà-Alabern. On the topology characterization of guifi. net. In Wireless and Mobile Computing, Networking and Communications (WiMob), 2012 IEEE 8th International Conference on, pages 389-396. IEEE, 2012.

[15] Davide Vega, Llorenç Cerdà-Alabern, Leandro Navarro, and Roc Meseguer. Topology patterns of a community network: Guifi. net. In Wireless and Mobile Computing, Networking and Communications (WiMob), 2012 IEEE 8th International Conference on, pages 612-619. IEEE, 2012.

[16] Guifi.net Growth Map website. http://guifi.net/en/guifi/menu/stats/growthmap.

[17] MikroTik Traffic-Flow documentation. http://wiki.mikrotik.com/wiki/Manual:IP/Traffic_Flow. 